\title{
Clinical outcome and risk factors for recurrence in borderline ovarian tumours
}

\section{Y Yokoyama*, , T Moriya ${ }^{2}$, T Takano ${ }^{3}$, T Shoji ${ }^{4}$ O Takahashi ${ }^{5}$, K Nakahara $^{6}$, H Yamada $^{7}, \mathrm{~N} \mathrm{Yaegashi}^{3}$,

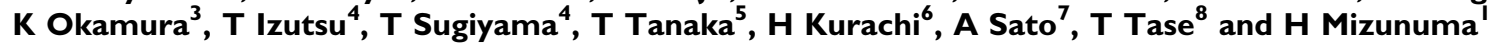

'Department of Obstetrics and Gynecology, Hirosaki University School of Medicine, 5 Zaifu-cho, Hirosaki 036-8562, Japan; '2Department of Pathology, Tohoku University School of Medicine, I-I Seiryo-cho, Aoba-ku, Sendai 980-8574, Japan; ${ }^{3}$ Department of Obstetrics and Gynecology, Tohoku University School of Medicine, I-I Seiryo-cho, Aoba-ku, Sendai 980-8574, Japan; ${ }^{4}$ Department of Obstetrics and Gynecology, Iwate Medical University School of Medicine, 19-I Uchimaru, Morioka 020-8505, Japan; ${ }^{5}$ Department of Obstetrics and Gynecology, Akita University School of Medicine, I-I I Hondo, Akita 0 10-8543, Japan; 'Department of Obstetrics and Gynecology, Yamagata University School of Medicine, 2-2-2 lidanishi, Yamagata 990-9585, Japan; ${ }^{7}$ Department of Obstetrics and Gynecology, School of Medicine Fukushima Medical University, I Hikarigaoka, Fukushima 960-I 295, Japan;

${ }^{8}$ Department of Gynecology, Miyagi Cancer Center, 47-I Aishima, Natori 98I-I293, Japan

We investigated the long-term prognosis of borderline ovarian tumours and determined risk factors for recurrence. One hundred and twenty-one borderline ovarian tumours treated between 1994 and 2003 at the participating institutions in the Tohoku Gynecologic Cancer Unit were retrospectively investigated for clinical stage, histopathological subtype, surgical technique, postoperative chemotherapy, the presence or absence of recurrence, and prognosis. The median follow-up period was 57 months ( I - 126 months). One hundred and nine cases (90.6\%) were at clinical stage I. The histopathological subtypes consisted of 9 I cases of mucinous tumour (75.2\%), 27 cases of serous tumour (22.3\%), and three cases of endometrioid tumour. Conservative surgery was used in 53 cases (43.8\%), radical surgery in 68 cases (56.2\%), a staging laparotomy in 43 cases (35.5\%), and postoperative adjuvant therapy in 30 cases (24.8\%). Recurrence was found in eight cases, but no tumour-related deaths were reported. Although no significant difference in disease-free survival rate was seen between different clinical stages, the difference in disease-free survival rate between serous and nonserous (mucinous and endometrioid) types was significant $(P<0.05)$. The I0-year disease-free survival rate was $89.1 \%$ for the radical surgery group and $57.4 \%$ for the conservative surgery group - this difference was significant $(P<0.05)$. In the conservative surgery group, cystectomy and serous tumour were independent risk factors for recurrence. Although recurrence was observed, the long-term prognosis of borderline ovarian tumour was favourable, without tumour-related deaths. Considering the favourable prognosis, conservative surgery can be chosen as far as the patient has a nonserous tumour and receive adnexectomy. However, in cases of serous type and/or receiving cystectomy special care should be given as relative risk rates of recurrence elevate by 2 -4-folds.

British Journal of Cancer (2006) 94, I586- I59|. doi:10.1038/sj.bjc.6603।39 www.bjcancer.com

Published online 9 May 2006

(c) 2006 Cancer Research UK

Keywords: borderline ovarian tumour; conservative surgery; cystectomy; serous tumour; multivariate analysis

Taylor (1929) found that some epithelial ovarian tumours showed clinically intermediate behaviour between benign and malignant, and called them 'semimalignant'. The International Federation of Gynecology and Obstetrics (FIGO) has formally introduced this concept as 'carcinoma of low malignant potential' in 1971, and the World Health Organization (WHO) as 'borderline tumour' in 1973, when the histological diagnostic criteria was proposed. The concept of borderline ovarian tumours was histologically defined as a disease entity that had been proposed clinically, and the adequacy of this histological definition has been repeatedly verified clinically.

*Correspondence: Dr Y Yokoyama;

E-mail:yokoyama@cc.hirosaki-u.ac.jp

Revised I 3 February 2006; accepted 3 I March 2006; published online 9 May 2006
With an accumulated experience and knowledge regarding the characteristics and management of borderline ovarian tumours, reclassification and redefinition have been attempted (Seidman and Kurman, 1996), and new prognostic factors have been proposed (de Nictolis et al, 1992; Gershenson et al, 1999). At present, many conflicting reports are causing confusion. As many of the patients are relatively young (Harris et al, 1992), preservation of fertility has been attempted with favourable results (Morice et al, 2001). However, there are also reports of recurrence or poor prognosis (Kaern et al, 1993; Gilks, 2003), and more precise prognostic factors are required. We believe that it is important to get a clear picture of the present status of borderline ovarian tumours, as it has been more than 30 years since the introduction of the concept of these tumours. Our retrospective multicentre study conducted an overall clinical analysis of borderline ovarian tumours. Our ultimate aim is to investigate the long-term prognosis of borderline ovarian tumours, and to determine the risk factors for recurrence. 


\section{MATERIALS AND METHODS}

Information on 124 patients with a diagnosis of epithelial borderline ovarian tumour who were treated at the Tohoku Gynecologic Cancer Unit consisting of eight institutes from 1994 to 2003 was collected using each institutional databases.

Central pathological review was adopted in this study. One of the authors reviewed 124 cases diagnosed by the gynaecological pathologists of each institutes concerning histologic typing and grading of the primary lesion and 121 cases of those were determined as an epithelial borderline tumour. The histopathologic criteria embodied in a recent conference with published commentaries (Bell et al, 2004; Ronnett et al, 2004), some of which are included in the current WHO classification of ovarian tumours (Tavassoli and Devilee, 2003) were used for the diagnosis of borderline ovarian tumours in this study. These tumours were staged according to FIGO criteria (1987).

Radical surgery was defined as hysterectomy with bilateral salpingo-oophorectomy. Conservative surgery was defined as any surgery that preserved the uterus and one or both ovaries. Conservative surgical procedure was performed as cystectomy or adnexectomy. Peritoneal cytology was performed systematically in both surgical procedures. Surgical staging in the present study was defined as including peritoneal cytology, omentectomy, and pelvic lymphadenectomy with or without paraaortic exploration (lymphadenectomy or biopsy or palpation), and peritoneal biopsy in radical or conservative surgery on occasion. These surgical procedures were performed depending on the surgical teams who provided the treatment and whether borderline tumour was diagnosed during or after the surgical procedure.

With regard to adjuvant chemotherapy, all women with advanced disease, those with stage Ic, and those with a likely persistence of residual tumour after cystectomy received platinumbased treatment in the early years of this study. Thereafter, chemotherapy was usually confined to women with advanced disease.

Comparisons of categorical variables were conduced by twotailed $\chi^{2}$ and Fisher's exact tests where appropriate. Evaluation of independent factors predicting disease-specific recurrence was conducted by nominal logistic regression analysis. Survival estimates were calculated using the Kaplan-Meier product limit method. Comparison between survival curves was made using the generalised Wilcoxon's test. Statistical significance was set at $P<0.05$. The patients who lost to follow-up were censored from the survival data.

Detailed information regarding patient's characteristics, treatment method, recurrence, and prognosis of the disease was abstracted from the medical record. We did not request institutional review board approval for this study because of its retrospective nature.

\section{RESULTS}

The median age of patients was 43 years old (range 15-76 years). Fifty-one patients $(42.1 \%)$ were below 40 years of age, and 29

Table I Clinical and histopathologic characteristics of patients with borderline tumours

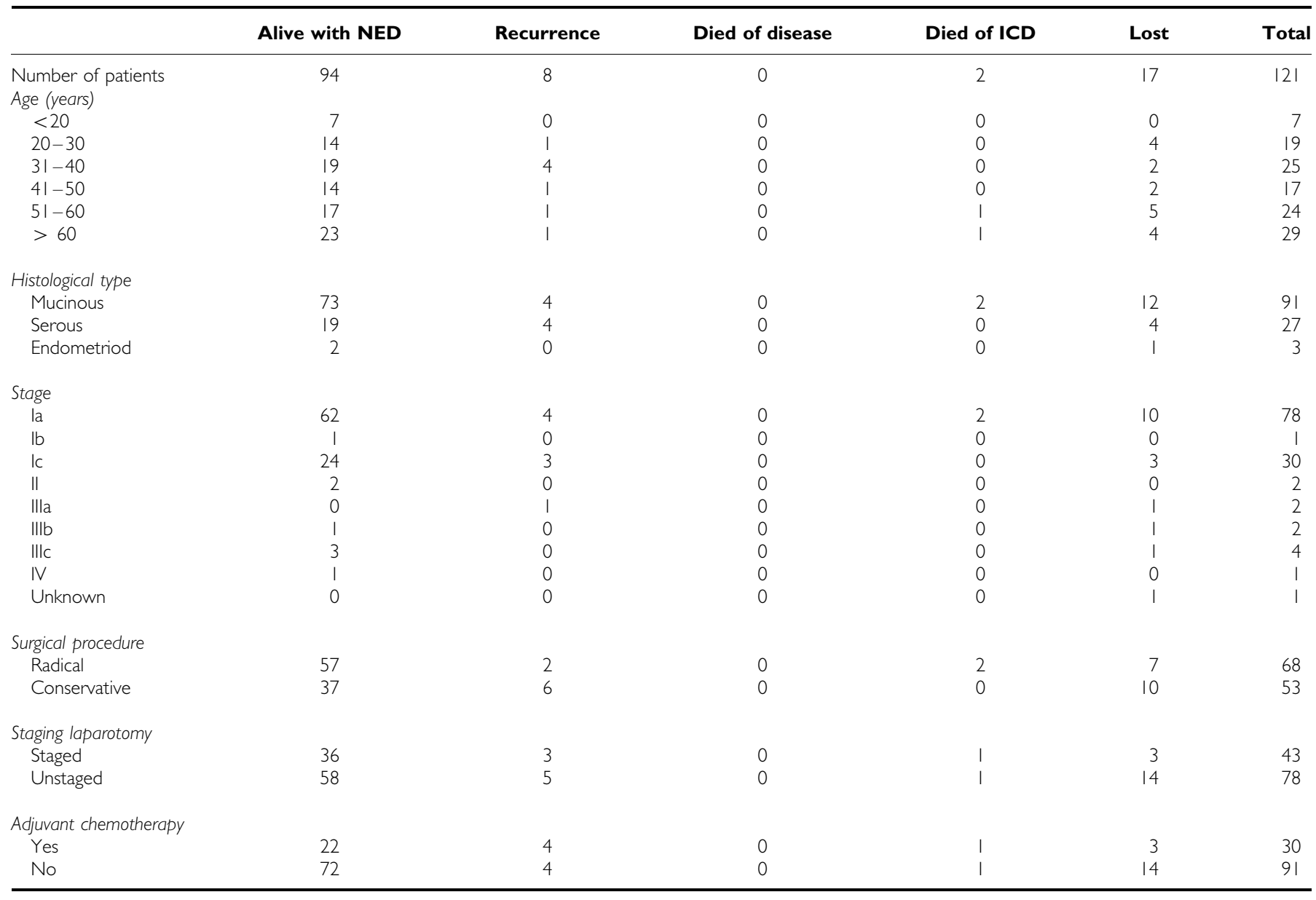

$\mathrm{NED}=$ no evidence of disease; $I C D=$ intercurrent disease. 
patients $(24.0 \%)$ were above 60 years of age (Table 1$)$. The followup period varied from 1 to 126 months, with a median of 57 months. One hundred and nine patients (90.6\%) had stage I disease, two had stage II disease, and nine had stage III and IV disease (Table 1). The dominant histopathological subtypes were mucinous (91 cases; $75.2 \%$ ) and serous (27 cases; $22.3 \%$ ) (Table 1 ). Only three tumours $(2.5 \%)$ were of endometrioid type. Seventyfive $(82.4 \%)$ and 16 of the 91 mucinous borderline tumours were intestinal and endocervical types, respectively. Radical treatment was performed in $68(56.2 \%)$ patients, and $53(43.8 \%)$ patients underwent conservative management (Table 1). Complete surgical staging was performed in $43(35.5 \%)$ patients (Table 1). Adjuvant chemotherapy was given to $30(24.8 \%)$ patients (Table 1$)$. Seventeen patients were lost to follow-up, and two patients died of the other diseases (Table 1). Four patients had a mucinous tumour with pseudomyxoma peritonei and were excluded from the present study because presence of pseudomyxoma peritonei changes the scope of management and the category of pseudomyxoma peritonei is recognised as tumour that can simulate primary mucinous borderline ovarian tumour (Ronnett et al, 2004).

Among 102 patients who were finally evaluated for clinical outcome and prognostic factors, eight had tumour recurrence but none of them died of the disease (Table 1). The median time to recurrence was $46 \pm 33$ months (range 14-107 months). The 5- and 10 -year disease-free survival rates were 91.7 and $69.2 \%$ for stage I diseases, respectively, and the 5- and 7-year disease-free survival rates were 100 and $66.7 \%$ for stage II-IV diseases, respectively (Figure 1A). The 10-year disease-free survival rate was 91.5 and $36.0 \%$ for mucinous and serous tumours, respectively (Figure 1B). Although no significant differences in disease-free survival rate were seen between different clinical stages, the difference between serous and nonserous (mucinous and endometrioid) types was significant. On the other hand, the 10-year disease-free survival rate was $89.1 \%$ for the radical surgery group and $57.4 \%$ for the conservative surgery group (Figure 1C). This difference was significant $(P<0.05)$. In univariate analysis, serous type and conservative surgery were found to be important variables affecting recurrence of disease (Table 2). Frequency of recurrence was not influenced by clinical stage, staging laparotomy, and postoperative adjuvant chemotherapy (Table 2). Multivariate analysis showed that only conservative surgery had independent prognostic value regarding recurrence of disease (Hazard ratio 2.2, $95 \%$ confidence interval, $0.02-0.52$ ) (Table 2). Subsequently, risk factors for recurrence were evaluated among 43 patients who underwent conservative surgery (Table 3 ). Of these patients, six had tumour recurrence (Table 3). Three of eight patients who had cystectomy and three of 35 patients who had adnexectomy experienced tumour recurrence (Table $3, P<0.03$ ). Recurrence occurred more frequently in patients with serous tumour than with nonserous tumour (Table 3). No correlation was found between recurrence and the factors such as clinical stage, staging laparotomy, or postoperative adjuvant chemotherapy among conservative surgery group (Table 3 ). Multivariate analysis confirmed cystectomy and serous type as an independent risk factor for recurrence of disease among the patients who underwent conservative surgery (Table 3 ). Table 4 shows estimated relative risk of having recurrence of disease for different combination of procedure of conservative surgery and histopathological subtype. For example, the relative risk for a patient receiving cystectomy for her serous tumour is 4.33 times greater than the risk for a patient receiving adnectomy for her nonserous tumour.

The clinical and pathological features of the eight patients who developed recurrence were demonstrated in Table 5. None of these eight patients died of progression of their disease. Three of the four serous tumours with recurrence were a noninvasive peritoneal implant, one of which was diagnosed as a serous adenocarcinoma at recurrence. The case developed adenocarcinoma in contralateral
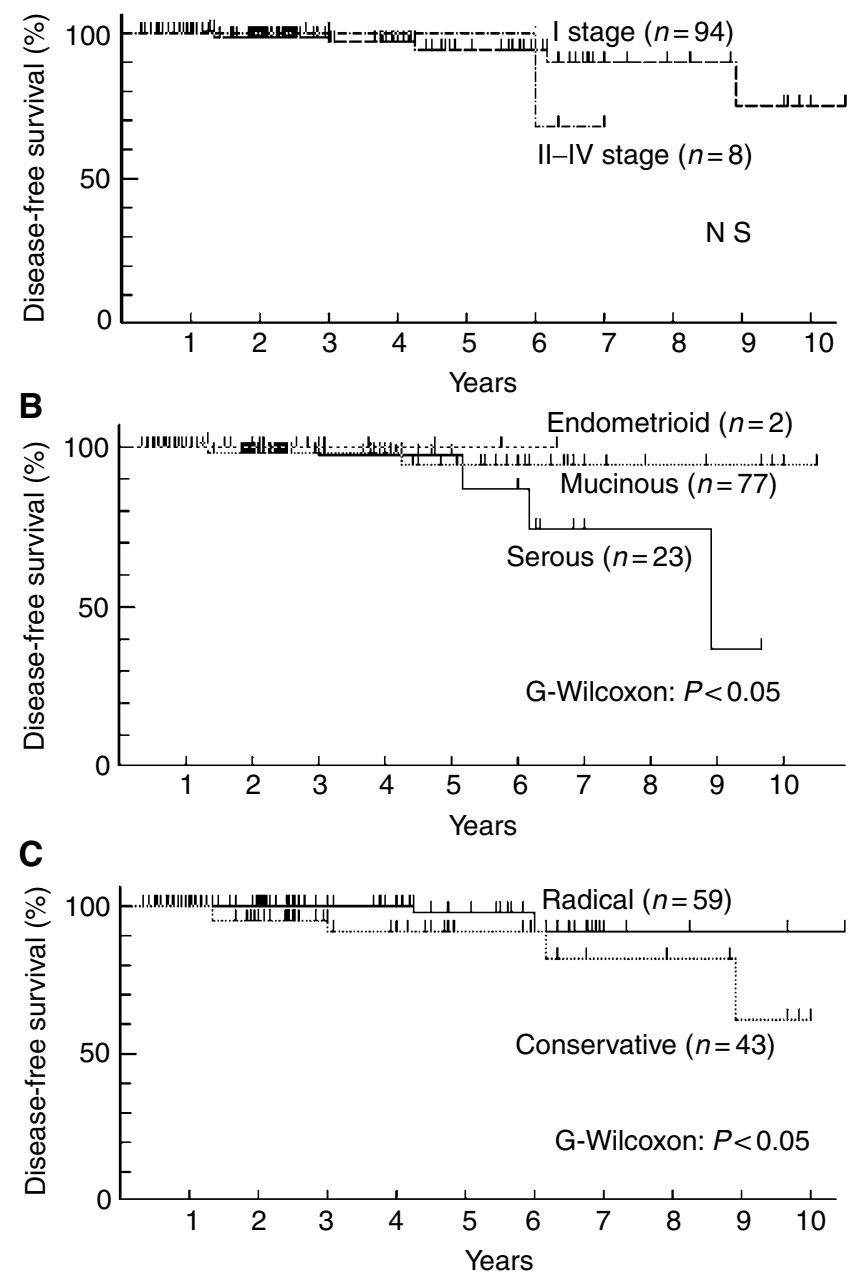

Figure I (A) Clinical stages and disease-free survival in patients with borderline ovarian tumour. There is no significant difference between two curves. (B) Histological type and disease-free survival in patients with borderline ovarian tumour. There is significant difference in disease-free survival between serous and nonserous (mucinous and endometrioid) type $(P<0.05)$. (C) Surgical procedure and disease-free survival in patients with borderline ovarian tumour. There is significant difference between two curves $(P<0.05)$.

ovary 107 months after cystectomy. All mucinous tumours with recurrence were of intestinal subtype. All patients with recurrence who were initially treated conservatively are free of disease after secondary surgical treatment.

\section{DISCUSSION}

It has been shown that the 5-year survival rate was $95-97 \%$ for stage I and $65-87 \%$ for stages II and III (Trope et al, 2000; Trimble et al, 2002; Sherman et al, 2004) suggesting that the prognosis for borderline ovarian tumours depends on extraovarian extension of the tumour. In addition, prognostic factors included clinical stage, histopathological subtype, and residual tumour, but the surgical method was not regarded as a prognostic factor (Trope et al, 2000; Gilks, 2003). The results of the present study, however, showed neither the stage nor the histopathological subtype of the disease was related with long-term prognosis, but showed that disease-free survival rates were significantly lower in cases managed by conservative surgery (Figure 1). 
Table 2 Risk factors for recurrence in borderline tumours

\begin{tabular}{|c|c|c|c|c|}
\hline Factors & $\begin{array}{l}\text { Recurrence } \\
\quad(n=8)\end{array}$ & $\begin{array}{c}\text { No recurrence } \\
\quad(n=94)\end{array}$ & $\begin{array}{c}\text { Univariate } \\
P\end{array}$ & $\begin{array}{c}\text { Multivariate } \\
P\end{array}$ \\
\hline $\begin{array}{l}\text { Mean age (years) } \\
\text { Histology, } n(\%)\end{array}$ & $42.2 \pm 13.7$ & $43.5 \pm 16.2$ & & \\
\hline Serous & $4(50)$ & $19(20.2)$ & & \\
\hline Nonserous & $4(50)$ & $75(79.8)$ & 0.053 & 0.09 \\
\hline \multicolumn{5}{|c|}{ Surgical procedure, n (\%) } \\
\hline Radical & $2(33.3)$ & $57(60.6)$ & & \\
\hline Conservative & $6(66.7)$ & $37(39.4)$ & 0.05 & 0.031 \\
\hline \multicolumn{5}{|c|}{ Staging laparotomy, n (\%) } \\
\hline Staged & $3(37.5)$ & $36(38.3)$ & & \\
\hline Unstaged & $5(62.5)$ & $58(61.7)$ & 0.96 & 0.58 \\
\hline \multicolumn{5}{|l|}{ Stage, n (\%) } \\
\hline I & $7(87.5)$ & $87(92.6)$ & & \\
\hline$I I-I V$ & I (I2.5) & $7(7.4)$ & 0.61 & 0.79 \\
\hline \multicolumn{5}{|c|}{ Adjuvant chemotherapy, n (\%) } \\
\hline Yes & $4(50)$ & $22(23.4)$ & & \\
\hline No & $4(50)$ & $72(76.6)$ & 0.098 & 0.33 \\
\hline
\end{tabular}

Table 3 Risk factors for recurrence in the patients who underwent conservative surgery for borderline tumours

\begin{tabular}{|c|c|c|c|c|}
\hline Factors & $\begin{array}{l}\text { Recurrence } \\
\qquad(n=6)\end{array}$ & $\begin{array}{l}\text { No recurrence } \\
\quad(n=37)\end{array}$ & $\begin{array}{c}\text { Univariate } \\
P\end{array}$ & $\begin{array}{c}\text { Multivariate } \\
P\end{array}$ \\
\hline \multicolumn{5}{|c|}{ Surgical procedure, n (\%) } \\
\hline Cystectomy & $3(50)$ & $5(13.5)$ & & \\
\hline Adnexectomy & $3(50)$ & $32(86.5)$ & 0.03 & 0.047 \\
\hline \multicolumn{5}{|c|}{ Staging laparotomy, n (\%) } \\
\hline Staged & (16.7) & $3(8.1)$ & & \\
\hline Unstaged & $5(83.3)$ & $34(91.9)$ & 0.51 & 0.137 \\
\hline \multicolumn{5}{|c|}{ Adjuvant chemotherapy, n (\%) } \\
\hline Yes & $3(50)$ & $7(18.9)$ & & \\
\hline No & $3(50)$ & $30(81.1)$ & 0.095 & 0.593 \\
\hline \multicolumn{5}{|l|}{ Stage, n (\%) } \\
\hline la & $3(50)$ & $23(62.2)$ & & \\
\hline lc & $3(50)$ & $14(37.8)$ & 0.57 & 0.198 \\
\hline \multicolumn{5}{|l|}{ Histology, n (\%) } \\
\hline Serous & $3(50)$ & $6(16.2)$ & & \\
\hline Non-serous & $3(50)$ & $31(83.8)$ & 0.059 & 0.041 \\
\hline
\end{tabular}

Table 4 Relative risk of recurrence in borderline tumours

\begin{tabular}{lcc}
\hline & \multicolumn{2}{c}{ Histological type } \\
\cline { 2 - 3 } Conservative surgery & Nonserous & Serous \\
\hline Adnexectomy & 1 & 2.11 \\
Cystectomy & 2.05 & 4.33 \\
\hline
\end{tabular}

In our study, surgical procedure was found to be an independent risk factor for recurrence and the risk could be reduced by radical surgery (Table 2). Because borderline tumours are seen more frequently in younger females than definitive carcinomas (Harris et al, 1992), whether conservative surgery is appropriate for

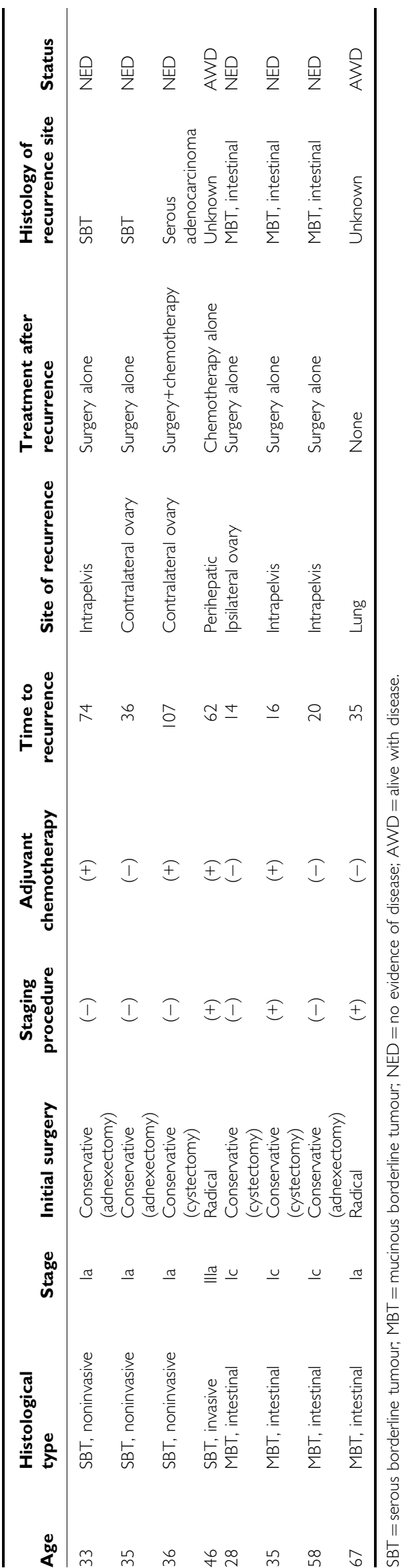

British Journal of Cancer (2006) $\mathbf{9 4}(\mid \mathrm{I}), \mid$ |586- |59| 
borderline ovarian tumours is an important matter to be resolved. Zanetta et al (2001) reported that only three of 119 stage I $(2.5 \%)$ cases that underwent radical surgery recurred, whereas 20 out of 164 stage I (12.1\%) cases that underwent conservative surgery recurred, with one case resulted in death from the disease. Morice et al (2001) demonstrated that the majority of recurrent cases, including stages II and III, were cured completely by subsequent surgery, and few cases resulted in death. More over, Donnez et al (2003) reported that although recurrence was commoner in cases treated by conservative surgery ( 3 out of $16,18.7 \%$ ) than by radical surgery (0 out of $59,0 \%)$, subsequent treatment resulted in no tumour-related deaths, and $63.6 \%$ of conservative surgery cases subsequently became pregnant, suggesting that conservative surgery can be an option for management of borderline malignant ovarian tumours in young subjects who need to reserve fertility. However, it is also reported that all of deaths as a result from recurrence were seen in cases treated by conservative surgery (Morris et al, 2000; Zanetta et al, 2001). Therefore, it is of quite importance to investigate underlying risk factors for recurrence after conservative surgery. As shown in Table 3, we found that cystectomy and serous tumours were independent risk factors for recurrence in patients who received conservative surgery. Previous reports have shown that recurrence after cystectomy did not necessarily occur ipsilaterally (Morris et al, 2000, 2001; Zanetta et al, 2001). So it seems that the residual tumour during cystectomy is solely responsible for recurrence. Then, it may be rational for young women who wish pregnancy to select cystectomy as an option if the surgical margin is free of tumour. However, results by Morice et al (2001) did not support this as they found that the recurrence rate was high after cystectomy compared with adnexectomy. Morris et al (2000) also demonstrated that recurrence was higher in cases treated by cystectomy rather than by adnexectomy. The present study confirmed this and further demonstrated for the first time that a difference in pathohistology affects the recurrence rate. As shown in Table 3, it was revealed that serous tumour is a significant risk factor for recurrence in cases managed by conservative surgery. Morris et al (2000) also showed similar tendency, but they regrettably missed statistical analysis. As shown in Table 4, the present study clearly demonstrated that the risk of recurrence when serous tumours were treated by cystectomy was approximately four times higher than for adnexectomy of nonserous tumours.

As a prognostic factor for borderline ovarian serous tumours, the concept of peritoneal implant is attracting attention (Bell et al, 1988). When estimating the prognosis of borderline ovarian serous tumours, peritoneal lesions should be explored and biopsied at the time of the surgery - in other words, accurate surgical staging is required. Clinical stage is one of the most important prognostic factors in borderline ovarian tumours (Kliman et al, 1986), and an accurate surgical staging is indispensable for follow-up after conservative surgery, as well as selecting postoperative therapy. Winter et al (2002) compared 48 cases that underwent complete surgical staging, and 45 cases without surgical staging - a higher stage was found in $17 \%$ ( 8 out of 48 ) of those assessed by surgical staging, but there was no difference in recurrence and survival rates between the groups. Camatte et al (2002) found metastasis to lymph nodes in $19 \%$ (8 out of 42 ) of cases. All cases with metastases were seen with borderline ovarian serous tumours associated with peritoneal dissemination, but no cases resulted in death - there was no difference in prognosis when compared with cases without metastases. The presence or absence of a peritoneal lesion is an important predictive factor of recurrence as well as an important prognostic factor, and we do not deny the importance of surgical examination of the abdominal cavity where possible. However, many reports have indicated that the presence or absence of lymph node metastasis is not related to the prognosis for borderline ovarian tumours (Camatte et al, 2002; Winter et al, 2002), and it is still debatable whether or not to perform a biopsy or dissection of the lymph nodes. As shown in Table 3, the present study could not show a significant relevance to risk of recurrence. The limitation of the present study is that surgical staging was not considered beforehand in all cases so that our data may be biased in this respect. Further studies using a prospective design with emphasis on surgical staging are required to investigate the risk of recurrence in borderline ovarian serous tumours after conservative surgery. Therefore, it is important that conservative surgery should only be performed in cases that truly require conservative surgery, after giving a full explanation of the risk of recurrence.

Barakat et al (1995) reported that cisplatin-based chemotherapy induced complete remission in six of $23(26 \%)$ advanced cases with macroscopic diseases, and in 17 of 25 (68\%) cases with microscopic disease, and proposed that adjuvant chemotherapy could be considered as a therapeutic option although a lifeextension effect of chemotherapy was not clear. In the present study, the regimen or frequency of chemotherapy used was not uniform, and differed among institutions, and no relationship was found between the presence or absence of postoperative adjuvant chemotherapy and recurrence (Table 2). Kaern et al (1993) showed that adjuvant chemotherapy did not improve neither recurrence free survival nor overall survival rate in 364 cases without residual tumour. Morice et al (2001) demonstrated that postoperative chemotherapy did not improve the survival rate in 80 cases of advanced borderline ovarian serous tumour in stages II and III with extraovarian extension, and that deaths were more closely related to the treatment than to the tumour. Thus, the efficacy of chemotherapy for borderline ovarian tumours is not yet established.

In conclusion, although recurrence was detected in eight out of 102 cases with borderline ovarian tumour that were available for follow-up, no tumour-related deaths were found, and there was a favourable long-term prognosis. Although the relative risk of recurrence is high, conservative surgery appears to be worth trying to preserve fertility, considering the favourable prognosis. When considering conservative surgery, special care should be given when cystectomy is chosen as a surgical procedure or the histological subtype is borderline serous ovarian tumour. Consensus has not been reached on such issues as to the significance of surgical staging, the indication for postoperative adjuvant chemotherapy, or the indications for conservative surgery. To reinforce the present study results, we expect that a large scaled prospective clinical study involving many institutions will be designed to obtain more evidence.

\section{REFERENCES}

Barakat RR, Benjamin I, Lewis Jr JL, Saigo PE, Curtin JP, Hoskins WJ (1995) Platinum-based chemotherapy for advanced-stage serous ovarian carcinoma of low malignant potential. Gynecol Oncol 59: $390-393$

Bell DA, Longacre TA, Prat J, Kohn EC, Soslow RA, Ellensosn LH, Malpica A, Stoler MH, Kurman RJ (2004) Serous borderline (low malignant potential, atypical proliferative) ovarian tumors: Workshop perspectives. Hum Pathol 35: 934-948

Bell DA, Weinstock MA, Scully RE (1988) Peritoneal implants of ovarian serous borderline tumors. Histologic features and prognosis. Cancer 62: $2212-2222$

Camatte S, Morice P, Atallah D, Pautier P, Lhomme C, Haie-Meder C, Duvillard P, Castaigne D (2002) Lymph node disorders and prognostic value of nodal involvement in patients treated for a borderline ovarian tumor: an analysis of a series of 42 lymphadenectomies. J Am Coll Surg 195: $332-338$ 
Donnez J, Munschke A, Berliere M, Pirard C, Jadoul P, Smets M, Squifflet J (2003) Safety of conservative management and fertility outcome in women with borderline tumors of the ovary. Fertil Steril 79: 1216-1221

Gershenson DM, Deavers M, Diaz S, Tortolero-Luna G, Miller BE, Bast Jr RC, Mills GB, Silva EG (1999) Prognostic significance of p53 expression in advanced-stage ovarian serous borderline tumors. Clin Cancer Res 5: $4053-4058$

Gilks CB (2003) Advanced stage serous borderline tumors of the ovary: A clinicopathological study of 49 cases. Int J Gynecol Pathol 22: 29-36

Harris R, Whittemore AS, Itnyre J (1992) Characteristics relating to ovarian cancer risk: collaborative analysis of 12 US case-control studies. III. Epithelial tumors of low malignant potential in white women. Collaborative Ovarian Cancer Group. Am J Epidemiol 136: 1204-1211

International Federation of Gynecology and Obstetrics (1987) Changes in definitions of clinical staging for carcinoma of the cervix and ovary. $A m \mathrm{~J}$ Obstet Gynecol 156: 263-264

Kaern J, Trope CG, Abeler VM (1993) A retrospective study of 370 borderline tumors of the ovary treated at the Norwegian Radium Hospital from 1970 to 1982. A review of clinicopathologic features and treatment modalities. Cancer 71: $1810-1820$

Kliman L, Rome RM, Fortune DW (1986) Low malignant potential tumors of the ovary: a study of 76 cases. Obstet Gynecol 68: 338-344

Morice P, Camatte S, El Hassan J, Pautier P, Duvillard P, Castaigne D (2001) Clinical outcomes and fertility after conservative treatment of ovarian borderline tumors. Fertil Steril 75: $92-96$

Morris RT, Gershenson DM, Silva EG, Follen M, Morris M, Wharton JT (2000) Outcome and reproductive function after conservative surgery for borderline ovarian tumors. Obstet Gynecol 95: 541 - 547

de Nictolis M, Montironi R, Tommasoni S, Carinelli S, Ojeda B, MatiasGuiu X, Prat J (1992) Serous borderline tumors of the ovary. A clinicopathologic, immunohistochemical, and quantitative study of 44 cases. Cancer 70: $152-160$

Ronnett BM, Kajdacsy-Balla A, Gilks CB, Merino MJ, Silva E, Werness BA, Young RH (2004) Mucinous borderline ovarian tumors: points of general agreement and persistent controversies regarding nomenclature, diagnostic criteria, and behavior. Hum Pathol 35: 949-960

Seidman JD, Kurman RJ (1996) Subclassification of serous borderline tumors of the ovary into benign and malignant types. A clinicopathologic study of 65 advanced stage cases. Am J Surg Pathol 20: 1331-1345

Sherman ME, Mink PJ, Curtis R, Cote TR, Brooks S, Hartge P, Devesa S (2004) Survival among women with borderline ovarian tumors and ovarian carcinoma: a population-based analysis. Cancer 100: 1045 - 1052

Tavassoli FA, Devilee P (2003) Tumours of the ovary and peritoneum. World Health Organization classification of tumours: Pathology and genetics of tumours of the breast and female genital organs. Oxford University Press: UK

Taylor HC (1929) Malignant and semi-malignant tumors of the ovary. Surg Gynecol Obstet 48: $701-712$

Trimble CL, Kosary C, Trimble EL (2002) Long-term survival and patterns of care in women with ovarian tumors of low malignant potential. Gynecol Oncol 86: 34-37

Trope CG, Kristensen G, Makar A (2000) Surgery for borderline tumor of the ovary. Semin Surg Oncol 19: 69-75

Winter 3rd WE, Kucera PR, Rodgers W, McBroom JW, Olsen C, Maxwell GL (2002) Surgical staging in patients with ovarian tumors of low malignant potential. Obstet Gynecol 100: 671-676

Zanetta G, Rota S, Chiari S, Bonazzi C, Bratina G, Mangioni C (2001) Behavior of borderline tumors with particular interest to persistence, recurrence, and progression to invasive carcinoma: a prospective study. J Clin Oncol 19: 2658-2664 